\title{
Medium chain triglycerides and hepatic encephalopathy
}

\author{
M. HILARY MORGAN, C. H. BOLTON, J. S. MORRIS ${ }^{1}$, AND A. E. READ \\ From the Department of Medicine, University of Bristol
}

SUMMARY The oral administration of short $\left(\mathrm{C}_{6}\right)$ and medium $\left(\mathrm{C}_{8}\right.$ and $\left(\mathrm{C}_{10}\right)$ chain triglycerides produced no clinical or electroencephalographic changes in patients with cirrhosis of the liver.

Arterial ammonia levels were also monitored in these patients and showed no significant change after medium chain triglycerides.

It was concluded that medium chain triglycerides, known to be of potential value in the treatment of malabsorption in patients with cirrhosis, are not clinically contraindicated, even in patients with evidence of hepatic encephalopathy.

Medium chain triglycerides which contain predominantly $C_{8}$ and $C_{10}$ saturated fatty acids have been found to be of value in the treatment of fat malabsorption due to a variety of causes including, for example, pancreatic insufficiency and intestinal disease (Fernandes, van de Kamer, and Weijers, 1955; French, McLeod, Chandler, Palm, and Porter, 1966) and liver disease (Burke and Danks, 1966).

In 1968 Linscheer confirmed that medium chain triglycerides were more readily absorbed than long chain triglycerides in patients with cirrhosis of the liver and fat malabsorption and that these substances gave rise to abnormally high serum levels of medium chain fatty acids.

Short and medium chain fatty acids have been reported to exhibit a narcotic effect on various species of experimental animals when given in large intravenous doses (Samson, Dahl, and Dahl, 1956; White and Samson, 1956). Also their relatively low affinity for serum albumin may permit increased penetration of the blood brain barrier (Boyer, Ballou, and Luck, 1947), especially in cirrhotic patients (Linscheer, Blum, and Platt, 1970). Because there is no clear evidence of any neurotoxicity in clinical trials in man this problem has been investigated in patients with chronic liver disease.

\section{Methods and Materials}

\section{PATIENTS}

A total of 21 patients with cirrhosis of the liver took part in the investigation. The pathological diagnoses ${ }^{1}$ Now at Department of Medicine, Royal Free Hospital, London. Received for publication 17 January 1974. were confirmed in all cases by biopsy and 11 patients had shown a tendency to develop hepatic encephalopathy, six in association with a previous porto-caval shunt operation.

\section{EXPERIMENTAL METHOD}

Eleven patients with cirrhosis of the liver and 11 informed volunteer control subjects fasted overnight and then received a single $60-\mathrm{ml}$ dose of coconut oil, administered as a $50 \%$ aqueous emulsion. Serum medium chain fatty acids, arterial ammonia levels, venous blood sugars, and EEGs were obtained before and at one and two hours after this loading dose. Venous blood sugar levels were monitored because of the increase in serum insulin levels which are known to follow ingestion of medium chain triglycerides (Linscheer, 1968). In five patients and two control subjects blood levels of medium chain fatty acids were monitored for four hours after 60 $\mathrm{ml}$ oral medium chain triglycerides, and in two of the patients blood levels were also monitored for 24 hours. In order to avoid obtaining erroneously high results for medium chain fatty acids due to the ingestion of other triglycerides during these 24-hour studies, an initial 12-hour fasting period was succeeded by a further 12 hours during which the patient was allocated a fat-free diet.

Twelve patients with cirrhosis and three volunteer control subjects received $30 \mathrm{ml}$ of coconut oil three times a day for one week and were monitored in a similar way before and after a week's treatment. Three of these patients underwent ammonia tolerance tests before and after the week's course of coconut oil. 


\begin{tabular}{|c|c|c|c|c|c|c|c|c|c|c|}
\hline \multirow[t]{2}{*}{ Patients } & \multicolumn{10}{|c|}{ Time in Hours } \\
\hline & 0 & 1 & 2 & 3 & 4 & 5 & 7 & 11 & 13 & 24 \\
\hline $\begin{array}{l}1 \\
2 \\
3 \\
4 \\
5\end{array}$ & $\begin{array}{l}0 \\
0 \\
0 \\
0 \\
0\end{array}$ & $\begin{array}{l}393^{1} \\
558 \\
215 \\
251 \\
453\end{array}$ & $\begin{array}{l}493 \\
798 \\
185 \\
447 \\
260\end{array}$ & $\begin{array}{l}393 \\
700 \\
177 \\
209 \\
195\end{array}$ & $\begin{array}{l}240 \\
732 \\
145 \\
167 \\
105\end{array}$ & $\begin{array}{l}335 \\
164\end{array}$ & $\begin{array}{l}60 \\
61\end{array}$ & $\begin{array}{lr}35 & 26 \\
\text { Not } & \text { measured }\end{array}$ & $\begin{array}{l}29 \\
65\end{array}$ & $\begin{array}{l}30 \\
37\end{array}$ \\
\hline
\end{tabular}

Table I Medium chain fatty acid levels in cirrhotic patients following $60 \mathrm{ml}$ oral medium chain triglycerides

${ }^{1}$ Levels in $\mu \mathbf{M}$

One patient with cirrhosis was studied before, during, and after receiving $30 \mathrm{ml}$ of coconut oil three times a day for three months, and a further patient remained on this dose for six months. Estimations of medium chain fatty acid levels in the cerebrospinal fluid after $30 \mathrm{ml}$ oral medium chain triglycerides were estimated in five control subjects and one cirrhotic patient who required a diagnostic lumbar puncture.

In three patients with cirrhosis the serum levels of hexanoic acid (MCF $\mathrm{C}_{6}$ ) were monitored after oral administration of glyceryltrihexanoate for 24 hours, and electroencephalographic recordings were also performed at one and two hours. The glyceryltrihexanoate was specially prepared and obtained from Lovelock, Sale, Manchester.

\section{BIOCHEMICAL ANALYSES}

Serum medium chain fatty acids

This method was based on the original Dole extraction procedure (Dole and Meinertz, 1960) followed by gas-liquid chromatography and utilizing an internal standard nonanoic acid. The internal standard used for the shorter chain $\left(C_{6}\right)$ fatty acid estimation was heptanoic acid.

\section{Arterial ammonia estimations}

Fasting blood ammonia was measured in arterial specimens by a modified method of Seligson and Hirahara (Seligson and Hirahara, 1957) in which a standard curve was constructed during each estimation. The percentage variation on a series of 20 standard estimations was plus or minus $16 \%$ at the 1.0 microgram level. Ammonia tolerance was estimated by measuring arterial ammonia levels at 45 and 90 minutes after an oral dose of $1 \mathrm{~g}$ of ammonium chloride, administered as a fruitflavoured drink.

\section{Electroencephalographic recordings}

The method used was that of Read and Laidlaw (Read, Laidlaw, and McCarthy, 1969) which allows calculation of the mean dominant frequency for each record. Values showing an inter-record variation of
$>0.6$ cycle are considered to be outside the normal cirrhotic day-to-day variation (Read, McCarthy, Ajdukiewicz, and Brown, 1968).

\section{Results}

Medium chain fatty acid levels in cirrhotic patients were monitored for four hours in five patients, and for 24 hours in two of these. The results are shown in table I, and confirm that there is rapid absorption, with maximum levels achieved within two hours, and also that serum levels fall again after seven hours. Further detailed studies were therefore restricted to evaluations over the first two hours, knowing that maximum medium chain fatty acid blood levels were achieved within this time, and that significant levels in the cerebrospinal fluid were also recorded over the same period of time (see table II).

\begin{tabular}{lll}
\hline Control 1 & $7 \cdot 5$ \\
2 & $2 \cdot 2$ \\
3 & $9 \cdot 3$ \\
4 & $9 \cdot 2$ \\
4 & $3 \cdot 4$ \\
5 & $9 \cdot 4$ \\
Patient & 1 & \\
(cirrhosis) & \\
\hline
\end{tabular}

Table II CSF levels of medium chain fatty acids ( $\mu M)$ one hr after $30 \mathrm{ml}$ medium chain triglyceride

FOLLOWING A SINGLE 60-ML DOSE OF COCONUT OIL

\section{EEG recordings}

There was no overall difference between the baseline and one- and two-hour figures for the mean dominant frequency following $60 \mathrm{ml}$ of medium chain triglycerides in 11 cirrhotic patients (fig 1), the mean change in dominant frequency at one hour being $+0.14 \pm 0.39$ and at two hours $+0.05 \pm 0.35$ cycles per second. Three of these patients with slow baseline records had previous encephalopathy. Six control subjects receiving $60 \mathrm{ml}$ of coconut oil were also found to be unaffected, the mean change in the EEG at one hour being $+0.21 \mathrm{cps}$ and at two hours $-0 \cdot 11$ cps. Two patients without encephalopathy 
EEG. RECORDINGS FOLLOWING $60 \mathrm{ml}$ MCT'S

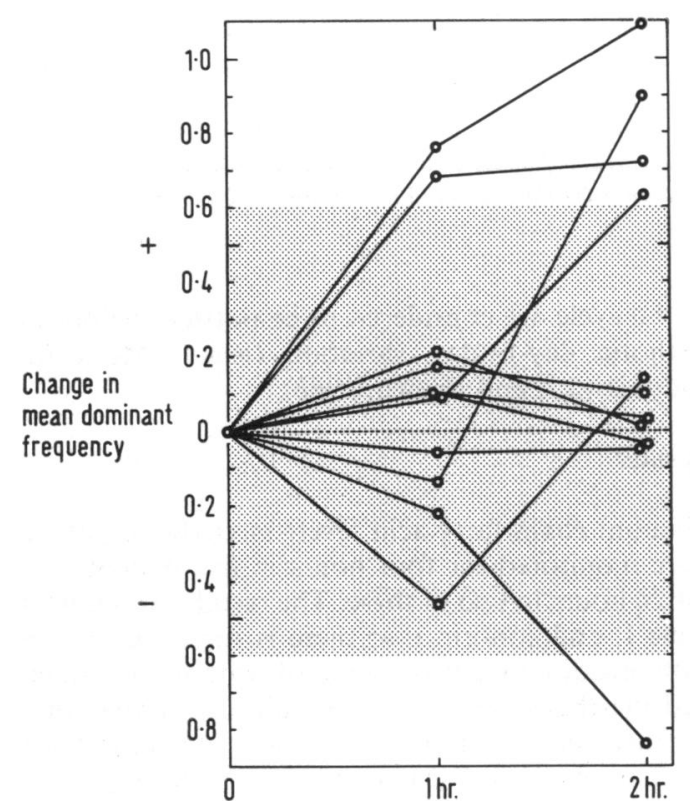

TOTAL SERUM MCF.A. CONCENTRATIONS

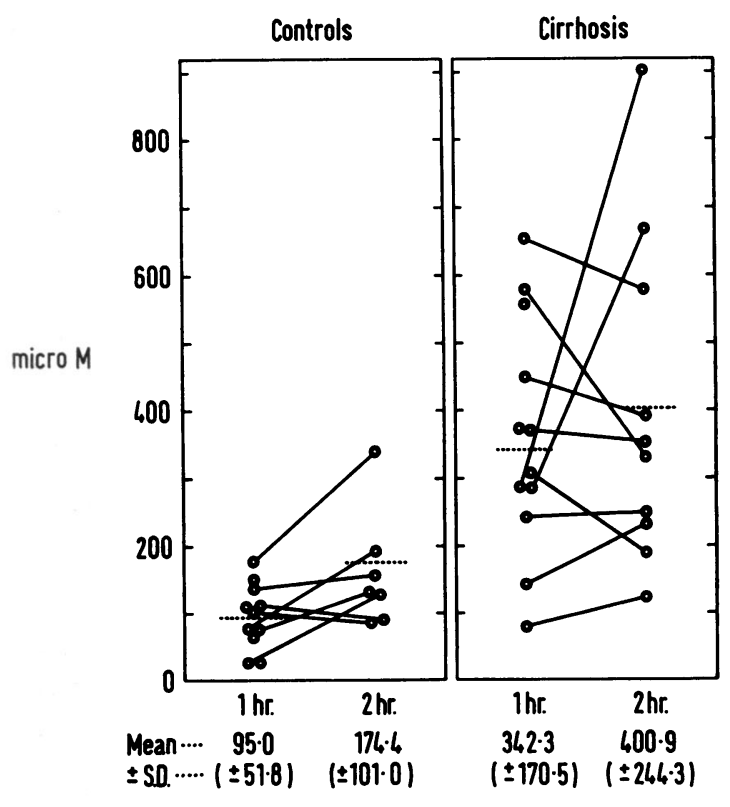

Fig 1 The change in mean dominant frequency of the EEG of patients with cirrhosis after $60 \mathrm{ml} \mathrm{MCT} \mathrm{orally.}$ EEG slowing is represented by negative values. The hatched area delineates the accepted range of day-to-day EEG variation in cirrhotic patients.

Fig 2 The serum medium chain fatty acid concentrations in controls and cirrhotic patients one and two hours after $60 \mathrm{ml}$ medium chain triglyceride orally.

who were monitored for 24 hours showed no significant change in their EEG mean dominant frequency.

\section{Biochemical results}

Venous blood sugar estimations before and at one and two hours after $60 \mathrm{ml}$ of medium chain triglycerides revealed no change. The results of similarly spaced arterial ammonia analyses were also unchanged in 10 of the 11 patients. The mean values were baseline $1.15 \pm 0.55$, one hour $1.03 \pm 0.51$, and two hours $1.20 \pm 0.55$. In one patient the arterial ammonia level at two hours was $0.53 \mu \mathrm{g}$ per $\mathrm{ml}$ higher than in the baseline analysis. However, the EEG was not significantly altered in this patient.

Medium chain fatty acid estimations

Figure 2 shows that in the group of cirrhotic patients the serum levels of medium chain fatty acids after a loading dose of $60 \mathrm{ml}$ medium chain triglyceride were approximately three times as high $(342 \cdot 3 \pm$ $170.5 \mu \mathrm{M})$ as the corresponding values in control subjects $(95.0 \pm 51.8 \mu \mathrm{M})$. In spite of the wide range of individual values in both groups the differences were statistically significant $(t=4.8057$ for one hour and $t=2.629$ for two hours, $P$ less than 0.01 ), but the differences between the one- and twohour values in the two groups were not significant.

\section{Chlorpromazine tolerance and medium chain triglycerides}

A single oral dose of medium chain triglyceride did not produce any significant change in the chlorpromazine tolerance exhibited by three cirrhotic patients, two of whom had had hepatic encephalopathy. There was no significant difference between the effect of chlorpromazine with and without $60 \mathrm{mg}$ medium chain triglyceride.

\section{RESULTS FOLLOWING MEDIUM CHAIN TRIGLYCERIDES FOR SEVEN DAYS (30 ML TDS)}

\section{Electroencephalographic recordings}

There was no significant difference in the EEG mean dominant frequency in cirrhotics or controls. 


\begin{tabular}{|c|c|c|c|c|c|c|}
\hline \multirow{2}{*}{\multicolumn{2}{|c|}{ Patient No. }} & \multicolumn{5}{|c|}{$C_{6}$ Serum levels $(\mu M)$ over 24 Hours } \\
\hline & & $\mathrm{OHr}$ & One $\mathrm{Hr}$ & $T w o H r$ & Six $H r$ & $24 \mathrm{Hr}$ \\
\hline
\end{tabular}

Table III Serial estimations of serum fatty acid levels $\left(C_{B}\right)$ after varying oral doses of the glyceryltrihexanoate

The mean change after one week was $+0.05 \pm 0.41$ for cirrhotic patients. In particular the EEGs of three patients with hepatic encephalopathy in this group also showed no significant change.

\section{Biochemical results}

Arterial ammonia estimations showed no significant change at the end of the treatment compared with the control week. The mean value in the control week was $1.29 \pm 0.46 \mu \mathrm{g} / \mathrm{ml}$ and after a week's therapy $1.24 \pm 0.49$.

Weekly liver function tests revealed no definite change during the administration of medium chain triglycerides. One icteric patient showed a mild increase in bilirubin level, from $11.5 \mathrm{mg} / 100 \mathrm{ml}$ to $15.3 \mathrm{mg} / 100 \mathrm{ml}$, but no parallel change in other parameters tested.

\section{MEDIUM CHAIN FATTY ACID ESTIMATIONS}

No significant serum levels of medium chain fatty acids were found in any of the patients who had been treated with medium chain triglycerides for a week, providing that the estimations were performed in the fasting state before the first morning dose.

\section{Results of investigations into the effect of the} medium chain triglyceride, glyceryltrihexanoate, on patients with cirrhosis

Twenty-four hour serum estimations of medium chain fatty 'acid $\left(\mathrm{C}_{6}\right)$ after oral medium chain triglyceride $\left(C_{6}\right)$ in single doses of $15 \mathrm{ml}, 30 \mathrm{ml}$, and $60 \mathrm{ml}$, respectively, showed that this fatty acid was rapidly absorbed and subsequently cleared from the blood. These results are shown in table III. No clinical change was observed in any of these three patients. Simultaneous EEG recordings at one and two hours showed an increased mean dominant frequency in two patients after $15 \mathrm{ml}$ glyceryltrihexanoate and in stored responses in the other two after 30 and $60 \mathrm{ml}$ respectively.

It was noted that absorption of the $\mathrm{C}_{6}$ was more rapid than of the $\mathrm{C}_{8}$ and $\mathrm{C}_{10}$ combination. Relative absorption rates following $30 \mathrm{ml}$ medium chain triglycerides in one patient with cirrhosis and hepatic encephalopathy can be seen in figure 3 .

\section{Discussion}

Following a single test dose of medium chain triglycerides, serum levels of medium chain fatty acids were found to be significantly higher in a group of cirrhotic patients than in a control group of noncirrhotic subjects, and these levels were rapidly achieved. Our results resemble those of Linscheer (Linscheer, 1970), and confirm his findings of relatively higher medium chain fatty acid levels in patients who had had a surgical shunt compared with

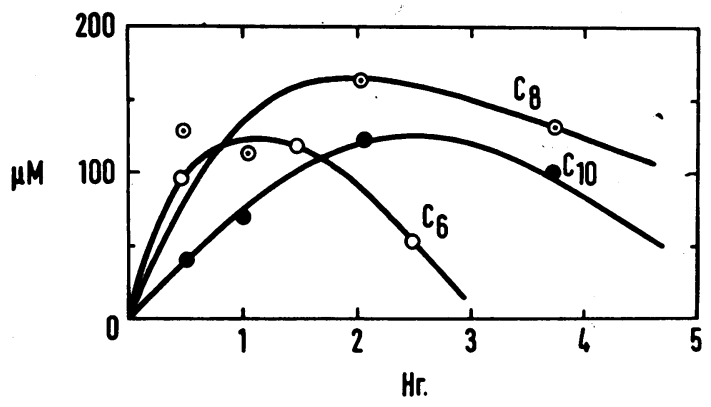

Fig 3 The blood levels of hexanoic acid $\left(C_{6}\right)$, octanoic acid $\left(C_{8}\right)$, and decanoic acid $\left(C_{10}\right)$ in a patient with hepatic cirrhosis and hepatic encephalopathy following $30 \mathrm{ml}$ of glyceryltrihexanoate and $30 \mathrm{ml}$ of medium chain triglyceride orally.

those who had not. This impaired hepatic clearance of medium chain fatty acid probably results from hepatic parenchymal dysfunction, as well as from the presence of portal systemic anastomoses (Linscheer, Patterson, Moore, Clermont, Robins, and Chalmers, 1966; Linscheer, Castell, and Platt, 1969).

The clinical significance of the presence of medium chain fatty acids in the cerebrospinal fluid is not clear (Linscheer, 1970). A possible role of medium and short chain fatty acids in the production of hepatic coma was suggested by Urabe and his associates following injections of butyric acid into dogs with hepatic insufficiency (Urabe, Tsubobawa, Ishiguro, Kiyosaki, and Iwasaki, 1964) and high levels of short and medium chain fatty acids are 
found in the systemic circulation of patients with hepatic encephalopathy (Zieve, 1966). Similar findings have also been reported with injections of short chain fatty acids in a variety of other experimental animals (Takahashi, Muto, Nakao, and Okinaka, 1966). Muto, in his work with rabbits (Muto, Takahashi, and Kawamura, 1964), reported drowsiness following intravenous octanoate, but the blood levels obtained were extremely high, though Clark (1968) reported narcosis following intestinal perfusion of medium chain triglycerides in rats.

No positive causal relationship between the degree of encephalopathy and the administration of medium chain triglycerides could be shown by Linscheer (1970), but the problem was not entirely resolved by this author. The present study has correlated clinical and EEG findings, and has shown no evidence of deterioration in clinical status, nor in EEG activity, following short or longer term administration of medium chain triglycerides, even in patients who had exhibited a previous tendency to develop encephalopathy. This does not necessarily disprove the hypothesis that very high serum levels of medium chain fatty acids might provoke some clinical change, but the oral ingestion of larger doses of medium chain triglycerides is precluded by gastrointestinal disturbances, such as nausea.

The possibility that the relative narcotic effect of different fatty acids could be related to chain length led to the comparison of medium chain fatty acids $\left(\mathrm{C}_{8}\right.$ and $\left.\mathrm{C}_{10}\right)$ with short chain fatty acids $\left(\mathrm{C}_{6}\right)$, but the only difference detected was slightly more rapid absorption and clearance of the latter substance.

While alterations in short chain fatty acid levels have been noted in patients with hepatic coma (Seegmiller, Schwartz, and Davidson, 1954; Thölen, Bigler, and Staub, 1961), no correlation between the elevation of these lipid substances and the presence and degree of altered consciousness has been obtained (Steigmann and Clowdus, 1971). The present findings also suggest that such a correlation is not of clinical importance, at least with therapeutic doses of medium chain triglycerides, and it may thus be argued that the presence of these fatty acids in the blood in increased quantities in liver failure is only a further reflection of the generalized alteration in hepatic metabolism.

The data presented have not confirmed suggestions that medium chain triglycerides may be contraindicated in patients with cirrhosis, and no clinical or neurological alteration was detected in the patients studied.
We are grateful to Miss J. Ford for technical assistance and to Dr J. Clamp for helpful discussions. Further technical assistance was given by Mrs D. Roberts and Mrs L. Arkley.

\section{References}

Boyer, P. D., Ballou, G. A., and Luck, J. M. (1947). The combination of fatty acids and related compounds with serum albumin. III. The nature and extent of the combination. J. biol. Chem., 167, 407-424.

Burke, V., and Danks, D. M. (1966). Medium chain triglyceride diet: its use in treatment of liver disease. Brit, med. J., 2, 1050-1051.

Clark, S. B. (1968). Limiting factors in maximal steady state absorption of medium chain triglycerides. In Medium Chain Triglycerides, edited by J. R. Senior, pp. 69-80. University of Pennsylvania Press, Philadelphia.

Dole, V. P., and Meinertz, H. (1960). Microdetermination of long chain fatty acids in plasma and tissues. J. biol. Chem., 235, 2595-1599.

Fernandes, J., Kamer, J. H. van de, and Weijers, H. A. (1955). Differences in absorption of the various fatty acids studied in children with steatorrhea. J. clin. Invest., 41, 488-494.

French, A. B., McLeod, G. M., Chandler, J. P., Palm, L. B., and Porter, C. (1966). The effect of medium-chain triglycerides (MCT) on losses of fat, nitrogen and minerals in patients with intestinal malabsorption. Amer. J.clin. Nutr., 18, 303-304.

Linscheer, W. G. (1968). Replacement of dietary fat by medium chain triglycerides in cirrhotic patients. In Medium Chain Triglycerides, edited by J. R. Senior, pp. 165-171. University of Pennsylvania Press, Philadelphia.

Linscheer, W. G., Blum, A. L., and Platt, R. R. (1970). Transfer of medium chain fatty acids from blood to spinal fluid in patients with cirrhosis. Gastroenterology, 58, 509-515.

Linscheer, W. G., Castell, D. O., and Platt, R. R. (1969). A new method for evaluation of portasystemic shunting: the rectal octanoate tolerance test. Gastroenterology, 57, 415-423.

Linscheer, W. G., Patterson, J. F., Moore, E. W., Clermont, R. J., Robins, S. J., and Chalmers, T. C. (1966). Medium and long chain fat absorption in patients with cirrhosis. J. clin. Invest., 45, 1317-1325.

Muto, Y., Takahashi, Y., and Kawamura, H. (1964). Effects of short chain fatty acids on the electrical activity of neo-, paleo-, and archicortical systems. Brain Nerve (Tokyo), 16, 608-611.

Read, A. E., Laidlaw, J., and McCarthy, C. F. (1969). Effects of chlorpromazine in patients with hepatic disease. Brit. med. $J$., $3,497-499$.

Read, A. E., McCarthy, C. F., Ajdukiewicz, A. B., and Brown, G. J. A. (1968). Encephalopathy after portacaval anastomosis. Lancet, 2, 999-1001.

Samson, F. E., Jr., Dahl, N., and Dahl, D. R. (1956). A study on the narcotic action of the short chain fatty acids. $J$. clin. Invest., 35, 1291-1298.

Seegmiller, J. E., Schwartz, R., and Davidson, C. S. (1954). The plasma 'ammonia' and glutamine content in patients with hepatic coma. J. clin. Invest., 33, 984-988.

Seligson, D., and Hirahara, K. (1957). The measurement of ammonia in whole blood erythrocytes and plasma. J. Lab. clin. Med., 49, 962-974.

Steigmann, F., and Clowdus, B. F., II. (1971). Hepatic Encephalopathy. Thomas, Springfield, Illinois.

Takahashi, Y., Muto, Y., Nakao, K., and Okinaka, S. (1966). Volatile fatty acids in hepatic coma. In Proceedings of the 3rd World Congress on Gastroenterology, 3, pp. 510-513.

Thölen, H., Bigler, F., and Staub, H. (1961). Zur Pathogenese des Urämie syndroms. I. Der Gehalt von Acetoin und 2,3-Butylenglykol im Blut uramischer Patienten. Path. et Microbiol. (Basel), 24, 262-269.

Urabe, M., Tsubobawa, T., Ishiguro, M., Kiyosaki, K., and Iwasaki, H. (1964). Study of the distribution of short-chain fatty acid in the brain of hepatic insufficiency dog. Jap. J. Gastroent., 1, 44-46.

White, R. P., and Samson, F. E., Jr. (1956). Effects of fatty acid anions on the electroencephalogram of unanesthetized rabbits. Amer. J. Physiol., 186, 271-274.

Zieve, L. (1966). Pathogenesis of hepatic coma. Arch. intern. Med. 118, 211-223. 